\title{
A Rare Reason of Cardiogenic Shock and Acute Myocardial Infarction: Aortic Valve Myxoma
}

\author{
Xianfeng Cheng, ${ }^{1,2}$ Dawei Shen, ${ }^{3}$ Jie Zhang, ${ }^{2}$ Tao Zhang ${ }^{4}$ \\ ${ }^{1}$ Department of Cardiac Surgery, Shandong Provincial Hospital, Cheeloo College of Medicine, Shandong University, Jinan, Shandong, China; \\ ${ }^{2}$ Department of Cardiovascular Surgery, Weifang People's Hospital, Weifang, China; \\ ${ }^{3}$ Department of Neurosurgery, Yidu Central Hospital of Weifang, Weifang, China; \\ ${ }^{4}$ Department of Cardiovascular Surgery, Shandong Provincial Hospital Affiliate to Shandong First Medical University, Jinan, China
}

\section{ABSTRACT}

Aortic valve myxoma is a rare benign cardiac neoplasm. The association of aortic valve myxoma with cardiogenic shock and acute myocardial infarction has been reported in few observations. We report the case of a 19-year-old male patient, who underwent chest pain for two weeks, then further examinations indicated a soft spherical mass on the left coronary cusp. The patient had sporadic cardiogenic shock and acute myocardial infarction during the preoperative preparation, and we carried out emergency effective cardiopulmonary resuscitation (CPR), followed by emergency surgical operation for aortic valve tumor.

Postoperative pathology showed it was a myxoma. The patient recovered smoothly and was discharged on postoperative day 7. Cardiogenic shock and acute myocardial infarction are very nonspecific, and we should be aware that patients with cardiogenic shock and acute myocardial infarction possibly suffer from aortic valve myxoma.

\section{INTRODUCTION}

Myxomas are the most commonly found cardiac primary tumors in adults. They are derived from multipotential mesenchymal cells located in the endocardium and can originate from cardiac structure or any chamber [Reardon 2003]. But most cardiac myxomas are located in the left atrium, attaching to the interatrial septum [Edvin 2015]. Myxomas arising from aortic valve are very infrequent [Wold 1980]. It is very rare in adolescents. Failure to settle accounts on time can result in a series of complications and sudden death [Edvin 2015].

We report the case of a patient with a stroke and an acute myocardial infarction who was found to have a mass on his aortic valve. The patient was treated surgically to prevent further ischemic complication. Subsequently, the histopathological examination confirmed the diagnosis of myxoma.

Received fune 3, 2021; accepted fuly 19, 2021.

Correspondence: Tao Zhang, Department of Cardiac Surgery, Shandong Provincial Hospital Affiliate to Shandong First Medical University, Fingwuweiqi Road 324, Finan 250021, China, Telephone +86-13606419191 (e-mail: zhangtaoshengli@163.com).

\section{CASE REPORT}

A healthy 19-year-old male without a background of cardiovascular disease developed chest pain for two weeks. The pain was compressive and not related with activity, but the onset of pain often was related to the change of posture. The physical examination, chest x-ray, and ECG were unremarkable, but the myocardial enzyme levels, including Troponin I, were elevated. The transthoracic echocardiogram revealed a soft spherical mass of $0.9 \times 1.2 \mathrm{~cm}$ in size on the left coronary cusp (LCC) of the aortic valve without aortic valve regurgitation. This medium echoic mass was displaced and moved in every cardiac cycle, and it was very close to the orifice of left coronary artery (LCA). Meanwhile, the muscle of the ventricular septum apical had poor mobility. The coronary computed tomograms (CT) also confirmed the existence of an aortic valve mass. (Figure 1)

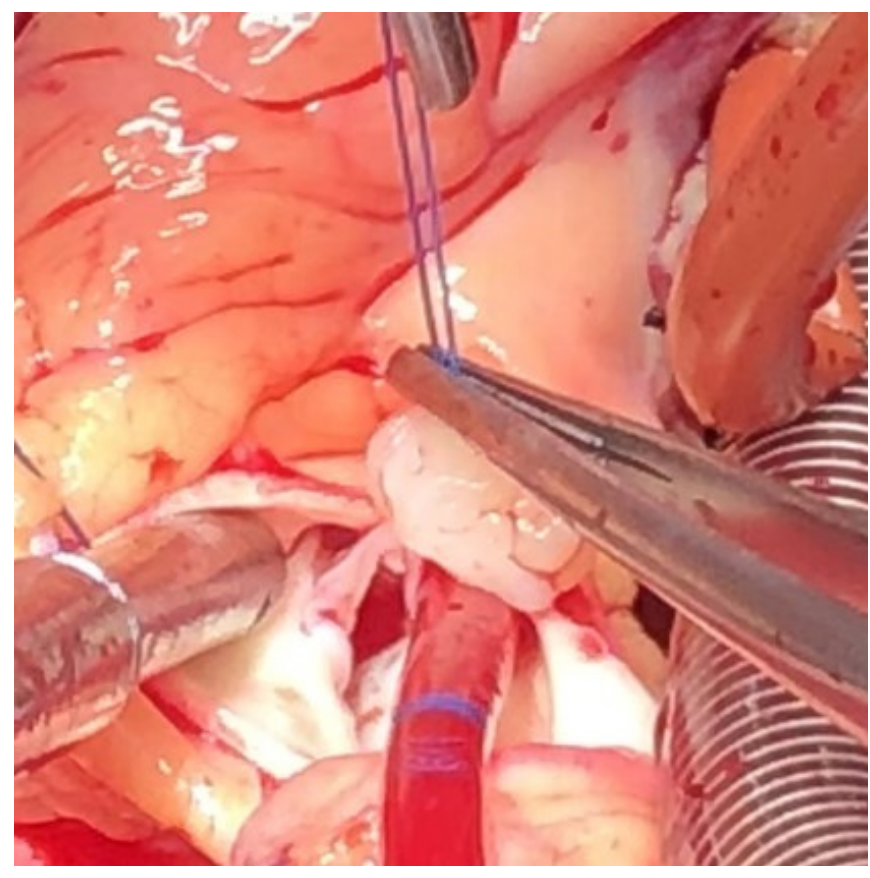

Figure 1. Echocardiography image and coronary computed tomograms image. Demonstrating a soft spherical mass on the left coronary cusp of aortic valve (arrows). 
The loss of consciousness and ventricular fibrillation of the patient suddenly occurred during the preoperative preparation, and emergency effective cardiopulmonary resuscitation (CPR) was carried out. After about two hours of CPR, he regained his regular pulse. The electrocardiogram showed acute myocardial infarction. (Figure 2) The patient underwent urgent surgical excision through a median sternotomy under cardiopulmonary bypass. A $0.8 \times 1.2 \mathrm{~cm}$ yellow-tancolored mass was attached to the left coronary cusp of the aortic valve. (Figure 3) This was observed to easily prolapse into the left coronary ostial. The mass successfully was shaved off and subsequently sent for pathology. A histopathological examination of the excised mass showed a paucicellular lesion contained oval and stellate cells with a moderate amount of eosinophilic cytoplasm, indistinct cell borders, and fine chromatin. (Figure 4) These features indicated it was a myxoma.

The postoperative echocardiogram showed no residual tumor and aortic valve regurgitation. The patient recovered smoothly and was discharged 7 days later. During the 6 months follow-up period, there was no evidence of local tumor recurrence.

\section{DISCUSSION}

Cardiac myxoma is a rare but not uncommon benign cardiac neoplasm. More than $75 \%$ of patients develop in the left atrium and only $15-20 \%$ of patients in the right atrium [Azevedo 2010], only $3 \%$ to $4 \%$ of myxomas are found in the left and right ventricle each [Reynen 1995]. Myxomas of the heart valves are rare, especially in the aortic valve. And meanwhile, a symptomatic manifestation in teenagers is extremely rare. Therefore, the difficulty of diagnosis of relevant patients is greatly increased.

As opposed to coronary heart disease, aortic valve myxoma is less strongly associated with cardiogenic shock and acute myocardial infarction, thus reducing the index of doubt about appearances. The nonspecific symptoms and findings of an obvious ST-segment elevation in the electrocardiogram indicate the potential difficulty in clinical diagnosis. Patients usually are misdiagnosed.

In the current case, our patient had acute myocardial infarction and cardiogenic shock before operation. It may be attributed to the aortic valve myxoma found protruding into the left coronary artery ostium. Therefore, clinicians should be conscious of acute myocardial infarction as a potential presentation of aortic valve myxoma.

Differential diagnosis of an aortic valve myxoma includes papillary fibroelastoma, Lambl's excrescences and vegetations [Edvin 2015]. Although histopathology remains the gold standard method of diagnosis [Fernandez 2012], the transthoracic echocardiogram or transesophageal echocardiography provides an economical and practical method for diagnosis [Zhenchun 2017]. And by analyzing ultrasound images, the size, shape, extension, attachment site and location, the mobility of a cardiac mass can promptly and easily be assessed, combining with the clinical symptom and medical history [Fernandez 2012].

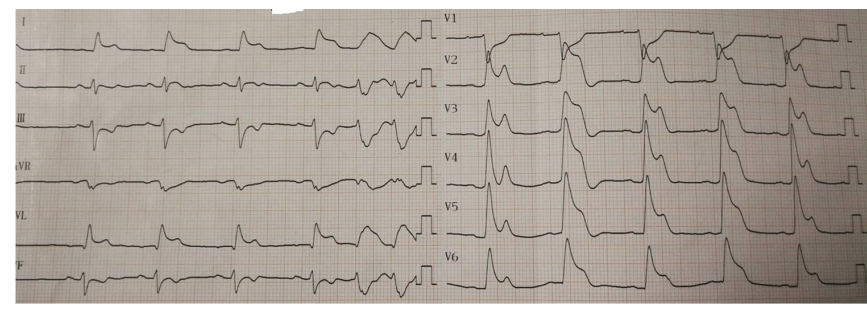

Figure 2. ECG showing diffuse saddle-shaped ST-segment elevation.

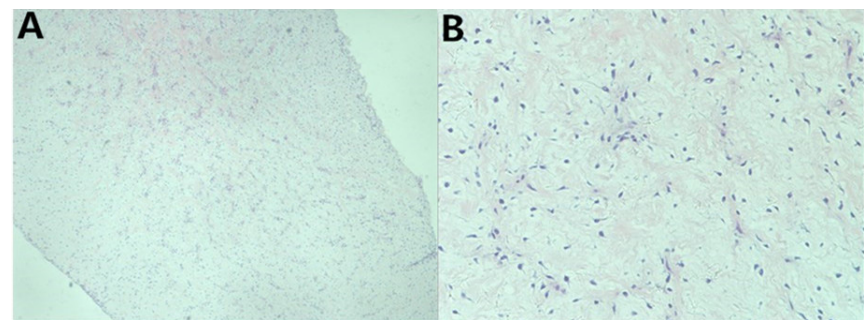

Figure 3. Surgical image, revealing the mass attached to the cusp of the left-coronary aortic leaflet.

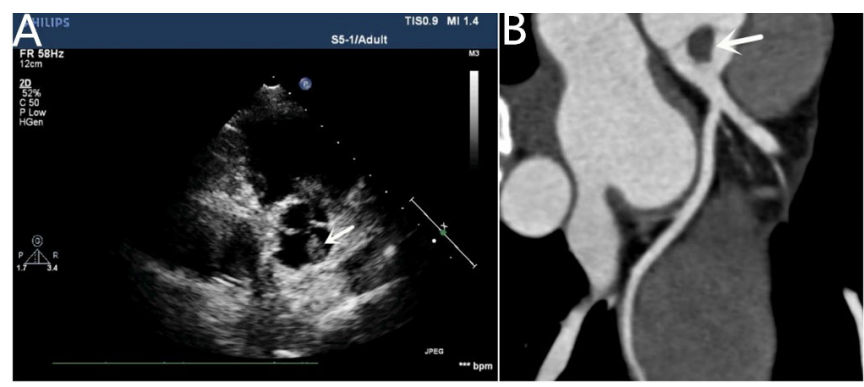

Figure 4. Histology sample. Haematoxylin and eosin stains at (A) $\times 40$ magnification showing a hypocellular lesion in a myxoid matrix and $(B)$ at $\times 200$ magnification showing oval cells and stellate with eosinophilic cytoplasm and indistinct cell borders.

Although the nonspecific symptoms or even occasional asymptomatic cases, what is undeniable is that aortic valve myxomas bring potential embolic issues [Zhenchun 2017]. Therefore, once diagnosed with aortic valve myxomas, surgical resection should be implemented as soon as possible. Surgical excision should contain the tumor and the implantation site to reduce the chance of tumors returning. And of course, the extent of surgery mainly depends on the size of the tumor. Although the long-term outcome of surgical excision of aortic valve myxomas is not fully understood due to its rarity, the recurrence rate appears very low. Therefore, rigorous follow up and monitor by echocardiography should be the basis for dealing with such cases.

\section{REFERENCES}

Azevedo O, Almeida J, Nolasco T, et al. 2010. Massive right atrial myxoma presenting as syncope and exertional dyspnea: case report. 


\section{Cardiovasc Ultrasound. 8:23.}

Edvin P, Fadil A, Efrosina K, et al. 2015. A giant myxoma originating from the aortic valve causing severe left ventricular tract obstruction: a case report and literature review. World J Surg Oncol. 13:151.

Fernandez AL, Vega M, El-Diasty MM. 2012. Myxoma of the aortic valve.

Interact Cardiovasc Thorac Surg. 15:560-2.

Reardon MJ, Smythe WR. 2003. Cardiac neoplasms. In: Cohn LH,
Edmunds LH(eds). Cardiac Surgery in the Adult. New York: McGrawHill. 1373-400.

Reynen K. 1995. Cardiac myxomas. N Engl J Med. 333:1610-7.

Wold LE, Lie JT. 1980. Cardiac myxomas: a clinico-pathologic profile. Am J Pathol. 101:219-33.

Zhenchun J, Longgang W, Jiacheng S, et al. 2017. Aortic Valve Myxoma in a Young Man: A Case Report and Review of Literature. Heart Surg Forum. 20:E066-E068. 